\title{
An Efficient Artificial Fish Swarm Model with Estimation of Distribution for Flexible Job Shop Scheduling
}

\author{
Hongwei Ge \\ College of Computer Science and Technology, Dalian University of Technology, \\ Dalian, 116023, China \\ Department of Computer Science and Engineering, Washington University in Saint Louis, \\ Saint Louis, Missouri, 63130, USA \\ E-mail:hwge@dlut.edu.cn \\ Liang Sun* \\ College of Computer Science and Technology, Dalian University of Technology, \\ Dalian, 116023, China \\ E-mail: liangsun@dlut.edu.cn \\ Xin Chen \\ College of Computer Science and Technology, Dalian University of Technology, \\ Dalian, 116023, China \\ E-mail: xinchendut@dlut.edu.cn \\ Yanchun Liang \\ College of Computer Science and Technology, Jilin University, \\ Changchun, 130012, China \\ E-mail: ycliang@jlu.edu.cn
}

Received 5 November 2015

Accepted 27 May 2016

\begin{abstract}
The flexible job shop scheduling problem (FJSP) is one of the most important problems in the field of production scheduling, which is the abstract of some practical production processes. It is a complex combinatorial optimization problem due to the consideration of both machine assignment and operation sequence. In this paper, an efficient artificial fish swarm model with estimation of distribution (AFSA-ED) is proposed for the FJSP with the objective of minimizing the makespan. Firstly, a pre-principle and a post-principle arranging mechanism that operate by adjusting machine assignment and operation sequence with different orders are designed to enhance the diversity of population. Following this, the population is divided into two sub-populations and then two arranging mechanisms are applied. In AFSA-ED, a preying behavior based on estimation of distribution is proposed to improve the performance of algorithm. Moreover, an attracting behavior is proposed to improve the global exploration ability and a public factor based critical path search strategy is proposed to enhance the local exploitation ability. Simulated experiments are carried on BRdata, BCdata and HUdata benchmark sets. The computational results validate the performance of the proposed algorithm in solving the FJSP, as compared with some other state of the art algorithms.
\end{abstract}

Keywords: Flexible job shop scheduling, artificial fish swarm model, estimation of distribution, Friedman test and Holm procedure.

\footnotetext{
* Corresponding author: College of Computer Science and Technology, Dalian University of Technology, Dalian, China. Tel: 86-41186980422, Email: liangsun@dlut.edu.cn.
} 


\section{Introduction}

The Flexible Job-shop Scheduling Problem (FJSP) is an extension of classical JSP for flexible production situation, which allows an operation to be processed by any machine from a given set. Generally, the FJSP can be divided into two sub-problems, i.e., the machine assignment problem that arranges each operation to a machine from a given set of alternative machines, and the operation sequence problem that determines the processing sequence of all operations to obtain a feasible schedule. It has been proved that FJSP is a strongly NP-hard problem ${ }^{1}$.

Many research efforts have focused on the development of efficient methods for FJSP. The first study was performed by Bruker and Schlie ${ }^{2}$ for two jobs FJSP, in which a polynomial graphical algorithm was developed. Then the researchers have concentrated on exact optimization techniques such as branch and bound $^{3,4}$, dynamic programming ${ }^{5}$, and disjunctive graph representation ${ }^{6,7}$. However, since the FJSP is a strongly nondeterministic polynomial-time hard problem, only moderate-size instances of the problems can be solved within a reasonable time by exact techniques. On the one hand, the approximate and heuristic methods make a tradeoff between solution quality and computational cost. These methods include dispatching priority rules ${ }^{8}$, shifting bottleneck approach ${ }^{9}$, and Lagrangian relaxation $^{10}$. More recently, with the emergence of new techniques from the field of artificial intelligence, much attention has been devoted to meta-heuristics. The tabu search (TS) has been widely used, such as Brandimart ${ }^{11}$, Mastrolilli and Gambardella ${ }^{12}$, Bozejko et al. ${ }^{13}$ and Li et al. ${ }^{14}$, while the genetic algorithm (GA) has also been examined to be an efficient method such as in Chen et al. ${ }^{15}$, Kacem et al. ${ }^{16}$, Pezzella et al. ${ }^{17}$ and Gao et al. ${ }^{18}$ Besides, some other meta-heuristics have been employed for this problem such as simulated annealing $(\mathrm{SA})^{19-21}$, particle swarm optimization $(\mathrm{PSO})^{22-24}$, ant colony optimization $(\mathrm{ACO})^{25}$, artificial neural network $(\mathrm{ANN})^{26}$, and artificial immune system (AIS) ${ }^{27}$.

Among the above algorithms, the meta-heuristics have acquired great achievements and become a popular tool for solving NP hard combinational optimization problems $^{28}$. The artificial fish swarm algorithm (AFSA) proposed by $\mathrm{Li}^{29}$ is a population-based meta-heuristic. It is insensitive to initial values, and possesses good performance such as fast convergence, high fault tolerance and robustness ${ }^{30}$. Thus it has gained an increasing study and wide applications such as multiobjective optimization $^{31}$, job shop schedule problem ${ }^{32}$ and clustering problem ${ }^{33}$. Motivated by these perspectives, we propose an efficient artificial fish framework with estimation of distribution (AFSA-ED) for FJSP. Meanwhile, some oriented heuristic strategies are proposed and embedded in the framework to enhance the overall performance, which include the integrated initialization process, the pre-principle and post-principle arranging mechanisms, the attracting behavior, and the public factor based critical path search strategy. The proposed algorithm balances the global exploration ability and the local exploitation ability.

\section{Flexible Job-shop Scheduling and Basic Artificial Fish Swarm Algorithm}

\subsection{Flexible Job-shop Scheduling Problem}

The FJSP can be described as follows. There are $n$ jobs $J=\left\{J_{1}, J_{2}, \cdots, J_{n}\right\}$ to be processed on $m$ machines $M=$ $\left\{M_{1}, M, \cdots M_{m}\right\}$. Each job $J_{i}$ has $n_{i}$ operations $\left\{O_{i, 1}, O_{i, 2}, \cdots\right.$, $\left.O_{i, n_{i}}\right\}$ to be processed according to a given sequence. Each operation $O_{i, j}$ can be processed on any machine among a subset $M_{i, j} \subseteq M$. The FJSP is to solve the assignment of machines and the sequence of operations to minimize a certain scheduling objective, e.g., the makespan of all the jobs $\left(C_{\max }\right)$.

Moreover, the following conditions should be satisfied while processing. Each machine processes one operation at a given time. Each operation is assigned to only one machine. Once the process starts, it cannot be interrupt. All jobs and machines are available at the beginning. The order of the operations for each job is predefined and cannot be modified.

\begin{tabular}{cllllll}
\multicolumn{6}{c}{ Table 1 } & A sample instance of FJSP \\
\hline Job & Operation & $M_{1}$ & $M_{2}$ & $M_{3}$ & $M_{4}$ \\
\hline \multirow{3}{*}{$J_{1}$} & $O_{1,1}$ & 3 & 5 & - & 6 \\
& $O_{1,2}$ & 6 & - & 4 & 5 \\
& $O_{2,1}$ & - & 5 & 2 & 3 \\
\multirow{3}{*}{$J_{2}$} & $O_{2,2}$ & 1 & 1 & 5 & 3 \\
& $O_{2,3}$ & 2 & 3 & - & 2 \\
\hline
\end{tabular}

For explaining explicitly, an example of FJSP is shown in Table 1 . There are 2 jobs and 4 machines, where the rows correspond to the operations and the 
columns correspond to the machines. Each element denotes the processing time of this operation on the corresponding machine, and the "-" means that an operation cannot be processed on the corresponding machine.

\subsection{Standard Artificial Fish Swarm Algorithm}

The artificial fish swarm algorithm (AFSA) is a population-based optimization algorithm, which is inspired from fish swarm behaviors. In an AFSA system, each artificial fish (AF) adjusts its behavior according to its current state and its environmental state, making use of the best position encountered by itself and its neighbors. The optimization of artificial fish swarm algorithm is conducted by four behaviors, i.e., preying, swarming, following, and moving.

Suppose $X_{i}=\left(x_{1}, x_{2}, \cdots, x_{n}\right)$ is the current position of artificial fish $A F_{i} ; Y_{i}=f\left(X_{i}\right)$ is the fitness function at position $X_{i}$. Visual is the visible distance of $A F$; try_number is the try times of preying behavior; Step is the maximum moving step of $A F ; \delta$ is the crowd factor; $n_{f}$ is the number of $A F \mathrm{~s}$ within its visual. For $A F_{i}$, one target position $X_{j}$ in its visual can be described by Eq.(1), Rand() is a function that generate random numbers in the interval $[0,1]$. Then the $A F_{i}$ updates its state by using Eq.(2) when the updating condition is satisfied.

$$
\begin{gathered}
X_{j}=X_{i}+\text { visual } \cdot \text { Rand }() \\
X_{i / \text { next }}=X_{i}+\frac{X_{j}-X_{i}}{\left\|X_{j}-X_{i}\right\|} \cdot \text { Step } \cdot \text { Rand }()
\end{gathered}
$$

The four behaviors of AF are described as follows:

(1) Preying: The $A F_{i}$ chooses a position $X_{j}$ randomly within its visible region using Eq.(1). If $Y_{j}<Y_{i}$, it moves one step to $X_{j}$ according to Eq.(2). Otherwise, it chooses another position and determines whether it satisfies the requirement $Y_{j}<Y_{i}$. If the requirement is still not satisfied after $t r y \_n u m b e r$ times, $A F_{i}$ executes the moving behavior.

(2) Swarming: Suppose $X_{C}$ is the center position in the visible region, if the center has more food and low crowd degree as indicated by $Y_{C} \cdot n_{f}<Y_{i} \cdot \delta$, then $A F_{i}$ moves a step towards $X_{C}$ according to Eq.(2). Otherwise, $A F_{i}$ executes default preying behavior.

(3) Following: Suppose $X_{b}$ is the best found position in the visible region, if the position $X_{b}$ has high food consistence and low crowd degree as indicated by $Y_{b} \cdot n_{f}<Y_{i} \cdot \delta$, then $A F_{i}$ moves a step towards $X_{b}$ according to Eq.(2). Otherwise, $A F_{i}$ executes default preying behavior.

(4) Moving: $A F_{i}$ choose a random position in its visual region and moves a step towards this direction. It is a default behavior of preying.

In AFSA, swarming and following are simulated in each generation. The AFs will choose the behavior to find the position with better fitness value, and the default behavior is preying. The flow chart of AFSA is shown in Fig. 1.

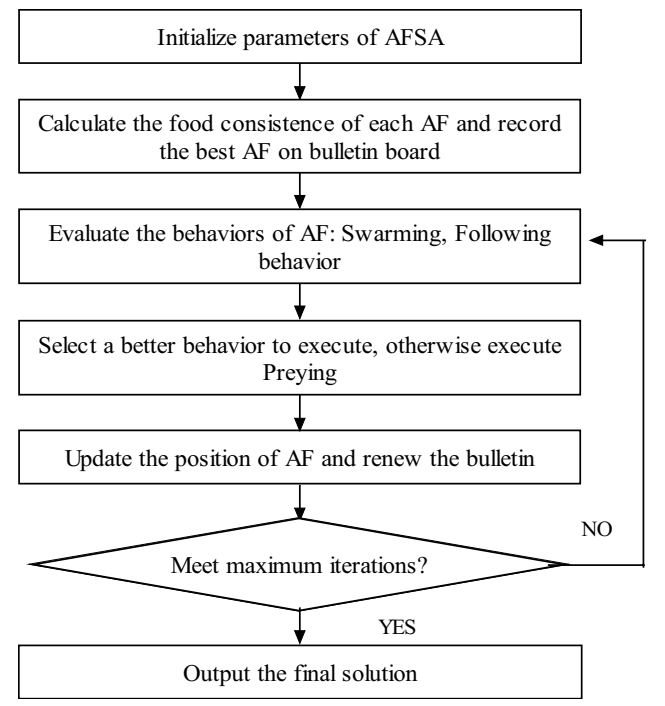

Fig.1 The framework of basic AFSA

\section{Artificial Fish Swarm Algorithm with Estimation of Distribution (AFSA-ED)}

The main components of the proposed AFSA-ED include the following strategies. Firstly, the preprinciple and the post-principle arranging mechanisms are applied to adjust the machine assignment and the operation sequence. Secondly, the preying behavior based on estimation of distribution is imitated by an object-oriented probability model. Thirdly, the attracting behavior is applied to improve the global search ability of algorithm. And finally, the local search based on critical path is applied to balance exploration and exploitation.

\subsection{Pre-principle and post-principle Mechanisms}

The FJSP optimizes the objective function by adjusting the machine assignment and the operation sequence. The order of solving the two sub-problems may affect the optimal results. Thus, we propose a pre-principle arranging mechanism and a post-principle arranging 
mechanism to adjust machine assignment and operation sequence. The objective is to enhance the diversity of population and make the algorithm searching the feasible solutions more comprehensively. The proposed mechanisms work as follows:

(1) Pre-principle arranging mechanism (PrA): The machine assignment part is firstly adjusted for balancing the workload of each machine, and then the operation sequence part is adjusted for minimizing the total makespan.

(2) Post-principle arranging mechanism (PoA): The operation sequence part is firstly adjusted for balancing the completion time of each job, and then the machine assignment part is adjusted for minimizing the total workload of machines.

While implementing the AFSA, the population is divided into two sub-populations. The two subpopulations respectively adopts the $\operatorname{PrA}$ and $\operatorname{PoA}$ mechanisms. After the independent evolution for each sub-populations, they are recombined to an entire population for further evolution.

\subsection{The preying behavior based on estimation of distribution}

In an AFSA system, preying behavior usually tends to be blindfold since the selection of destination locations is achieved by a random process. To overcome this shortcoming, we propose to estimate the distribution of the individuals, and then use the distribution model to guide preying behavior. The estimation of distribution algorithm (EDA) can reduce the randomness of behavior and make the search move toward and converge to the promising regions in the solution space.

The EDA works as follows: (1) Select a set of promising individuals from the population according to the fitness value; (2) Estimate the probability distribution of the selected individuals according to a probabilistic model. The probability distribution is constructed by two matrixes, i.e., the machine probabilistic matrix and the operation probabilistic matrix; (3) Generate new individuals according to the estimated probability.

Let $I$ denote the number of promising individuals selected from the current population, and $D_{1}^{l}$ be the machine probabilistic matrix at the $l$ th generation. Each entry $d_{i j k}^{l}$ of $D_{1}^{l}$ means the probability of operation $O_{i, j}$ being processed on machine $M_{k}$ and it is determined by the following formula:

$$
d_{i j k}^{l}=a_{i j k}^{l} / I
$$

Where $a_{i j k}^{l}$ is the number of individuals that select $M_{k}$ to process operation $O_{i, j}$.

Let $D_{2}^{l}$ denote the operation probabilistic matrix at the $l$ th generation. Each entry $d_{i j}^{l}$ of $D_{2}^{l}$ means the probability of job $J_{j}$ being arranged in position $i$ in the operation sequence and it is calculated by the following formula:

$$
d_{i j}^{l}=a_{i j}^{l} / I
$$

Where $a_{i j}^{l}$ is the number of individuals arranging $J_{j}$ in position $i$.

While implementing the preying behavior, a new individual is generated by sampling the two probabilistic matrixes. The machine assignment vector is generated through sampling the probabilistic matrix $D_{1}^{l}$. For each operation $O_{i, j}$, machine $M_{k}$ is selected with a probability of $d_{i j k}^{l}$. Similarly, the operation sequence vector is generated by sampling the probabilistic matrix $D_{2}^{l}$. Job $J_{j}$ is selected with a probability of $d_{i j}^{l}$ to replace the $j$-th position of operation sequence vector.

\subsection{Attracting behavior}

In AFSA, each $A F$ determines its next position according to current state and its environmental state within its visible region, which may limit the exploration ability and interaction with the other AFs outside its visible region. We propose an attracting behavior to enhance exploration ability.

The bulletin board that records the state of the current optimal individual is setup. For each AF, it reads the position information of the optimal individual from the bulletin board, and then it moves one step toward this direction.

Suppose $X_{i}$ is the current position of $A F_{i}, Y_{i}$ is the fitness value. $X_{b e s t}$ is the position of the global optimal individual and its fitness value is $Y_{\text {best }}$. If $Y_{i}>Y_{\text {best }}$, then $A F_{i}$, moves towards $X_{b e s t}$ for a step according to Eq. (2). Otherwise, if $Y_{i}=Y_{\text {best }}$, which means that it is the optimal individual, then it executes default preying behavior.

\subsection{Critical Path Local Search based on Public Factor}

In this subsection, a local search procedure is presented to enhance the exploitation around the best solution obtained by the AFSA. The local search is based on the 
critical path, so it is applied to the schedule represented by the disjunctive graph rather than by the AF. Hence, when a solution is to be improved by the local search, it should be firstly decoded to a schedule represented by the disjunctive graph.

\subsubsection{The disjunctive graph}

A feasible solution of FJSP can be represented by the disjunctive graph $G=(N, B \cup C)$, where $N$ is a set of nodes which includes all the operations and two dummy nodes: starting and terminating; $B$ is a set of conjunctive arcs which represents precedence constraints in the same job; $C$ is a set of disjunctive arcs which correspond to the precedence of operations processed on the same machine. The weight of each node is the processing time of corresponding operation. In a disjunctive graph, the longest path form the starting node to terminating node is called critical path, whose length determines the makespan of the schedule. Any operation on the critical path is called critical operation.

Take the problem shown in Table 1 for instance, a possible schedule represented by the disjunctive graph is showed in Fig.2. $S_{G}$ and $E_{G}$ are respectively dummy starting and terminating nodes. The operations $O_{1,1}$ and $O_{2,3}$ are performed on $M_{1}$ successively, $O_{2,1}$ and $O_{1,2}$ are performed on $M_{3}$ successively, and $O_{2,2}$ is processed in $M_{2}$.

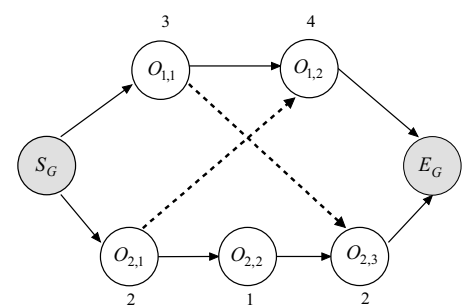

Fig.2 Illustration of framework of disjunctive graph

\subsubsection{Neighborhood structure based on public factor}

The disjunctive graph usually has more than one critical paths. Only changing the length of all the critical paths, the makespan can be changed. For obtaining a better schedule from the current one, lots of operations may be tried to move. The process is time consuming. So the public factor based critical path search in the neighborhoods is proposed. The public factor is used to identify the influence degree of the critical for all the critical paths. The public factor of an operation $O_{i, j}$ can be defined by the formula:

$$
P f_{i, j}=N_{i, j} / T_{c p}
$$

Where $T_{c p}$ is the total number of critical paths, and $N_{i, j}$ is the number of the critical paths including the operation $O_{i, j} \cdot P f_{i, j}$ is in the interval $[0,1] . P f_{i, j}=$ 0 means this operation does not include any critical path, in other words, it is not a critical operation; $P f_{i, j}=1$ means this operation is included in all critical paths. For each critical operation, the higher value of public factor one operation possess, the greater impact for disjunctive graph it has while moving it. In the neighborhood structure, the critical operation with the highest public factor will be moved preferentially.

While moving an operation, the precedence constrains should be satisfied. For an operation $O_{i, j}$ processed on $M_{k}$, we define $S E_{G}\left(O_{i, j}\right)$ as the earliest start time and $C E_{G}\left(O_{i, j}\right)=S E_{G}\left(O_{i, j}\right)+t_{i, j, k}$ as the earliest completion time. Similarly, denote the latest start time without delaying the makespan as $S L_{G}\left(O_{i, j}\right)$ and the latest completion time as $C L_{G}\left(O_{i, j}\right)=S L_{G}\left(O_{i, j}\right)+t_{i, j, k}$. Let $P J_{i, j}=O_{i, j-1}$ be the precedent of operation $O_{i, j}$ and $F J_{i, j}=O_{i, j+1}$ be the successor of operation $O_{i, j}$. Denote $P M_{i, j}$ as the operation performed on $M_{k}$ right before $O_{i, j}$ and $S M_{i, j}$ as the operation performed on $M_{k}$ right after $O_{i, j}$. In the disjunctive graph $G$, the process of moving an operation $O_{i, j}$ is to delete it from its current machine sequence by moving all its disjunctive arcs and then insert it at another available machine by adding disjunctive arcs. Let $P O_{l}(l=1,2, \cdots, x)$ be the critical operation to be moved, where $x$ is the total number of critical operations in $G$. Let $G^{-}$be the disjunctive graph obtained by deleting the critical operation $P O_{l}$ from $G$. For no increasing the makespan after inserting operation $P O_{l}$, we take $C_{\max }(G)$ as the makespan of $G^{-}$when we calculate the latest start time $S L_{G^{-}}\left(O_{i, j}\right)$ for each operation in $G^{-}$. If $P O_{l}$ is inserted before $O_{i, j}$ on machine $M_{k}$ in $G^{-}$, it could be started as early as $C E_{G^{-}}\left(P M_{i, j}\right)$ and should be completed as late as $S L_{G^{-}}\left(O_{i, j}\right)$ without delaying the required makespan $C_{\text {max }}(G)$. In addition, $P O_{l}$ needs to comply with the operation precedence constraints. So, the available idle time for inserting $P O_{l}$ to machine $M_{k}$ need to satisfy the following condition:

$$
\begin{gathered}
\max \left\{C E_{G^{-}}\left(P M_{i, j}\right), C E_{G^{-}}\left(P J_{P O_{l}}\right)\right\}+t_{P O_{l}, k} \\
<\min \left\{S L_{G^{-}}\left(O_{i, j}\right), S L_{G^{-}}\left(F J_{P O_{l}}\right)\right\}
\end{gathered}
$$

This moving process is repeated until a better schedule strategy is found or all critical operations have been tried to move. The procedure of local search is shown in Algorithm 1. 


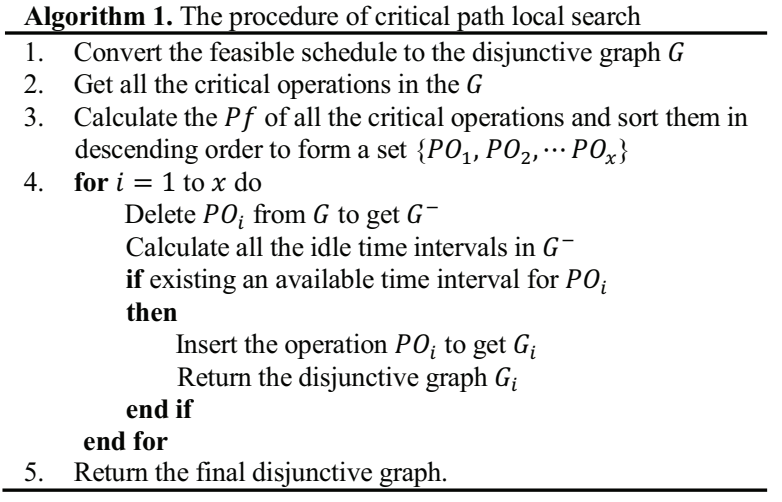

Figure 3 gives an illustration of the local search for the example in Table 1. In Fig.3 (a), the only critical path is $S_{G} \rightarrow O_{1,1} \rightarrow O_{2,1} \rightarrow O_{2,2} \rightarrow O_{2,3} \rightarrow E_{G}$, and the makespan is 17. The critical operation set is $P O_{l}=$ $\left\{O_{1,1}, O_{2,1}, O_{2,2}, O_{2,3}\right\}$, and all the public factors for the critical operations are 1 . So we can try to move the critical operations $O_{1,1}, O_{2,1}, O_{2,2}$, and $O_{2,3}$ successively. In this process, the algorithm preferentially selects the machine with the least processing time is and then judges the feasibility according to Eq. (6). Fig.3 (b) show the disjunctive graph $G_{1}$ obtained by moving the critical operation $O_{1,1}$ to machine $M_{1}$. In this case, the makespan is reduced to 12 . Fig.3 (c) show the disjunctive graph $G_{2}$ obtained by moving the critical operation $O_{2,1}$ to machine $M_{3}$. IN this case, the makespan is 11 . Following this, the critical operation $\mathrm{O}_{2,2}$ is not satisfied the moving condition according to Eq. (6). Finally, the algorithm obtains the disjunctive graph Fig.3 (d) by moving the critical operation $O_{2,3}$ to machine $M_{4}$. In this case, the makespan is reduced to 9 .

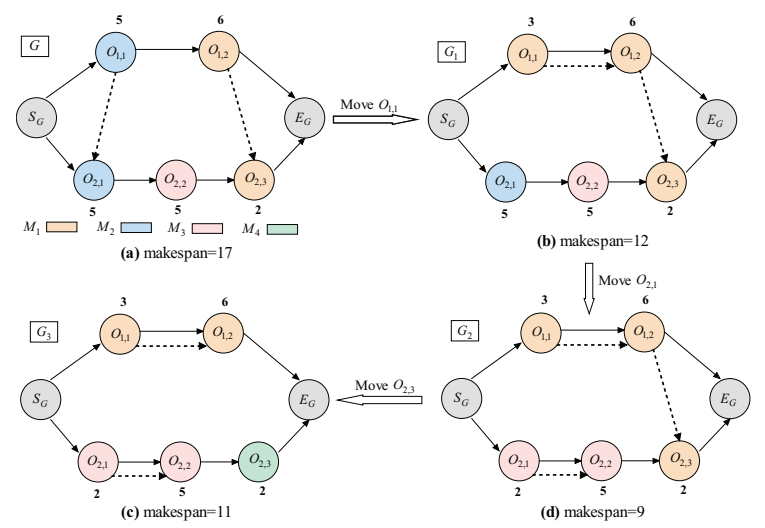

Fig.3 Illustration of the local search

\section{The Implementation of AFSA-ED for FJSP}

In this section, we will give the implementation of the AFSA-ED for FJSP. Firstly, the representation of the
AF, decoding method and the population initialization are introduced. Then, the framework of the algorithm is presented.

\subsection{Representation and movement}

In AFSA-ED, each AF represents a feasible solution of the problem. Each AF is expressed by two vectors: machine assignment vector and operation sequence vector, which correspond to the two sub-problems of the FJSP. The machine assignment vector is represented by a vector of $N$ integer values and $N$ is the total number of operations. Each element of vector denotes the machine selection of each operation and the value is the index of the array of alternative machine set. The operation sequence vector is an un-partitioned permutation with $n_{i}$ repetitions of job $J_{i}(i=1,2, \cdots, n)$. The length of operation sequence vector equals to $N$. The index $i$ of job $J_{i}$ occurs $n_{i}$ times in the vector, and the $k$-th occurrence of a job number refers to the $k$-th operation in the technological sequence of this job.

For the problem in Table 1, a representation of a feasible solution is shown in Fig.4. The machine assignment vector is $(\mathbf{1}, 2,2,2,3)$. If the operation $O_{2,1}$ is processed on $\left\{M_{2}, M_{3}, M_{4}\right\}$, then the corresponding element ' 2 ' means that operation $O_{2,1}$ will be assigned to the second machine $M_{3}$. If the operation sequence is given as $(2,1,2,2,1)$, then scanning the vector from left to right, the processing order of operations can be obtained: $O_{2,1}-O_{1,1}-O_{2,2}-O_{2,3}-O_{1,2}$.

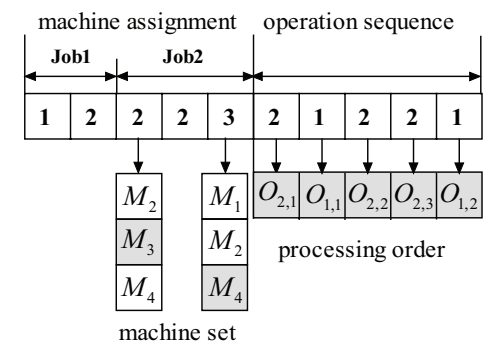

Fig.4 Illustration of the representation of a solution

To address the discrete FJSP, the movement of an AF in the solution space is completed by learning the partial structure from the target AF. Fig. 5 gives an illustrative example of the movement process. Assume the $i$-th AF is $(1,2,2,2,3 ; 2,1,2,2,1)$ in the current generation, and its target $\mathrm{AF}$ is $(2,2,1,3,2 ; 1,2,2,1,2)$, then an indicator vector with the same length is produced by randomly filled with the elements of the set $\{0,1\}$. The number of the element " 1 " is $\operatorname{int}\left[\frac{N}{2}\right]$ in the first part of the indicator vector, whereas the second part of the indicator vector has only one position filled by " 1 ". The elements in the 
first part of the indicator vector decide the sources of the corresponding elements in new produced $\mathrm{AF}$, namely from the parent or the target. The elements " 1 " in the second part of the indicator vector represents the starting position of the operation sequence segment from the target $\mathrm{AF}$, and its length is also taken as $\operatorname{int}\left[\frac{N}{2}\right]$, and the remainder is from the parent $\mathrm{AF}$. Finally, $m$ machine selections and operation orders are adjusted randomly according to the corresponding update formula, and $m=\operatorname{int}[\operatorname{step} \cdot \operatorname{rand}()]$ as in the formula (2).

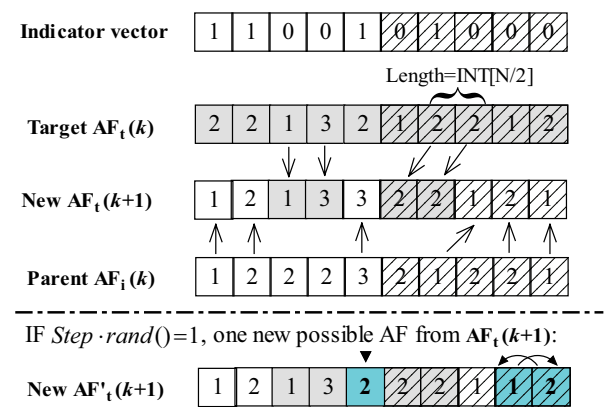

Fig.5 Illustration of the movement of an AF

\subsection{Decoding of $A F$}

For calculating the value of the makespan, each individual in the artificial fish swarm is decoded to the corresponding schedule sequence. The decoding is achieved by the process that assigns operations to the machines at their earliest possible starting time according to technological order of the jobs. It is worth noticing that the scheduling acquired in this way is semi-active. Then the active decoding is applied, which checks the possible blank time interval before appending an operation at the last position, and fills the first blank interval before the last operation to convert the semi-active schedule to an active one so that the makespan can be shorten ${ }^{34}$.

\subsection{Initialization}

In this subsection, an integrated initialization algorithm is proposed for machine assignment initialization and operation sequence initialization. To generate the initial machine assignments, the following rules are applied:

(1) Global approach of localization (GAL) ${ }^{17}$.

(2) Local approach of localization (LAL).

(3) Random rule.

The LAL makes a random permutation for the positions of machines. Following this, for each operation, it selects the machine with minimum processing time in the alternative machine set, and updates the machine workload by adding this processing to the processing time of the remaining unarranged operations within the same job. Take the problem showed in Table 1 as an example, Table 2 shows a possible machine assignment obtained by using the LAL. The last four columns indicate the final assignments obtained by LAL. In the table, the items in bold type are the updated workload of machines, and the "-" means that the operation cannot be processed on the corresponding machine.

The random rule executes by randomly selecting a machine from the alternative machine set for each operation. The GAL emphasizes the global workload among all the machines. The advantage of the LAL is that it obtains different initial assignments in different runs of the algorithm and emphasizes the workload among the set of machines within the same job. In addition, the random rule can increase the diversity of initial population. In our algorithm, the above three rules are used in a hybrid way. More specially, the initial machine assignments of $30 \%$ solutions in the population are generated by the GAL, $50 \%$ solutions by the LAL, and $20 \%$ solutions by random rule.

In our algorithm, the initial operation sequences are generated by the following three dispatching rules:

(1) Most time remaining (MTR). The job with the most remaining processing time will be arranged first.

(2) Most number of operations remaining rule (MOR). The job with the most remaining unprocessed operations has a high priority to be arranged.

(3) Random rule. Randomly generate the sequence of the operations. In particular, the above three rules are used in a hybrid way, then $20 \%$ of initial operation sequences are generated by random rule, $40 \%$ by the MTR, and $40 \%$ by the MOR.

Table 2 Initial assignments by LAL

\begin{tabular}{cccccccccccccccccc}
\hline & $M_{3}$ & $M_{2}$ & $M_{4}$ & $M_{1}$ & $M_{3}$ & $M_{2}$ & $M_{4}$ & $M_{1}$ & $M_{3}$ & $M_{2}$ & $M_{4}$ & $M_{1}$ & $\ldots$ & $M_{3}$ & $M_{2}$ & $M_{4}$ & $M_{1}$ \\
\hline$O_{1,1}$ & - & 5 & 6 & 3 & - & 5 & 6 & 3 & - & 5 & 6 & 3 & $\ldots$ & - & 5 & 6 & 3 \\
$O_{1,2}$ & 4 & - & 5 & 6 & 4 & - & 5 & 9 & 4 & - & 5 & 9 & $\ldots$ & 4 & - & 5 & 9 \\
$O_{2,1}$ & 2 & 5 & 3 & - & 2 & 5 & 3 & - & 2 & 5 & 3 & - & $\ldots$ & 2 & 5 & 3 & - \\
$O_{2,2}$ & 5 & 1 & 3 & 1 & 5 & 1 & 3 & 1 & 5 & 1 & 3 & 1 & & 7 & 1 & 3 & 1 \\
$O_{2,3}$ & - & 3 & 2 & 2 & - & 3 & 2 & 2 & - & 3 & 2 & 2 & & - & 4 & 2 & 2 \\
\hline
\end{tabular}




\subsection{The framework of AFSA-ED}

The framework of AFSA-ED for solving FJSP is shown in Fig.6. At the beginning of each generation, the entire population is randomly divided into two equal size subpopulations with one sub-population using machine based mechanism, and the other sub-population using operation based mechanism. Each AF evaluates by executing swarming, following, attracting behaviors. If the algorithm obtains a better solution using the three behaviors, it selects a better behavior to execute, otherwise, it executes the default improved preying behavior. When all individuals complete the searching process, the two sub-populations are combined into an entire population. Then the optimal individual of the population executes the critical path based on local search for further exploitation. If a new better individual is obtained, then the bulletin board is updated accordingly. The algorithm stops when the maximum iteration time is reached.

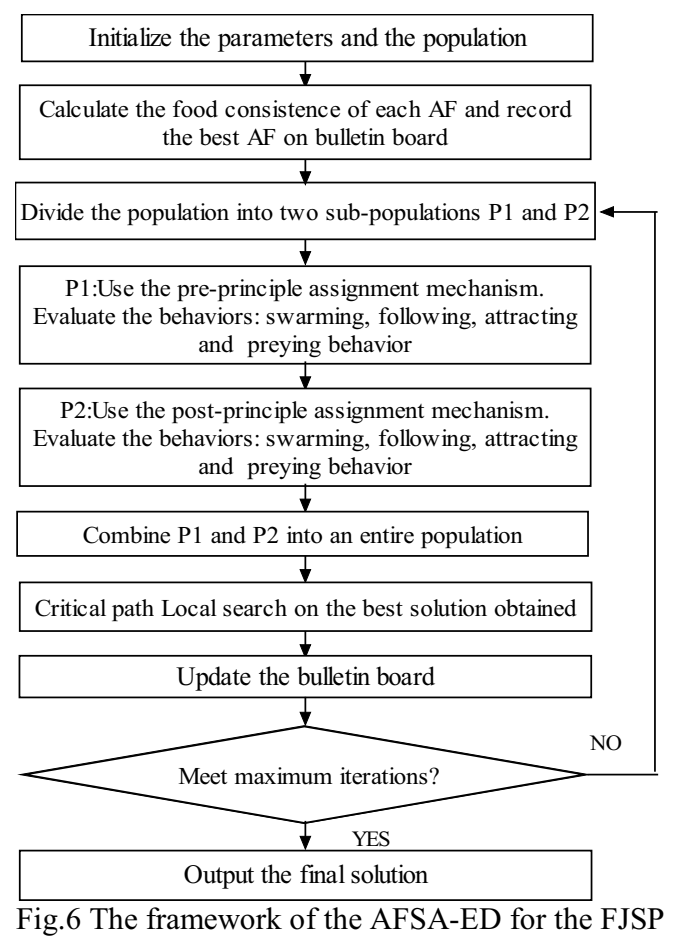

\section{Experimental Results}

\subsection{Instances and parameters}

To evaluate the performance of the AFSA-ED, we consider three sets of well-known benchmarks with 160 instances:
(1) BRdata: The data set includes 10 instances from Brandimarte $^{11}$. The number of jobs ranges from 10 to 20 , the number of machines ranges from 4 to 15 , and the flexibility of each operation ranges from 1.43 to 4.10 .

(2) BCdata: The data set includes 21 instances from Barnes and Chambers ${ }^{35}$, which were acquired from the classical JSP mt10 and the la24, la40 instances. The number of jobs ranges from 10 to 15 , the number of machines ranges from 11 to 18 , and the flexibility of each operation ranges from 1.07 to 1.30 .

(3) HUdata: The data set includes 129 instances from Hurink et al. ${ }^{36}$, which were obtained from 3 classical JSP instances (mt06, mt10, mt20) by Fisher and Thompson and 40 classical JSP instances (la01-la40) by Lawrence. HUdata is divided into three subsets: Edata, Rdata, and Vdata. The number of jobs ranges from 6 to 30 , the number of machines ranges from 5 to 15 , and the flexibility of each operation ranges from 1.15 to 7.5.

The AFSA-ED is coded and implemented in matlab language on an Intel Core i5 $2.53 \mathrm{GHz}$ personal computer with $1 \mathrm{~GB}$ of RAM. The algorithm runs 30 independent times for each instance from BRdata and BCdata, and runs 10 independent times for each instance from HUdata on account of the large number of instances in this data set. The computational results are compared with several performing algorithms from the existing literatures.

Each instance can be characterized by the following parameters: number of jobs $(n)$, number of machines $(m)$, number of operations $\left(T_{0}\right)$ and the flexibility of problem (Flex). The parameters in the AFSA-ED include population size $(A F S)$, the maximum iteration times, the try number, the step of AF (Step), the visual of AF (Visual), and the crowd factor (delta). In our experiment, the iteration times and the try number are taken as 40. The settings of the other parameters for each data set are listed in Table 3.

\begin{tabular}{|c|c|c|c|c|}
\hline Data set & $A F S$ & Step & Visual & delta \\
\hline BRdata & 20 & 5 & 40 & 10 \\
\hline BCdata & 40 & 10 & 100 & 20 \\
\hline HUdata & 60 & 20 & 200 & 30 \\
\hline
\end{tabular}

\subsection{Computational results}

The computational results for each data set are shown in this subsection. In the following tables, (LB,UB) denotes the lower and upper bounds ${ }^{37}$. The LB of BRdata and BCdata instances are taken from Mastrolilli 12, while the LB of HUdata instances are computed by Jurisch. ${ }^{38} C_{m}$ denotes the best makespan. $A V$ denotes the average makespan. $S D$ denotes standard deviation of the 
makespan. $T_{a}$ is the average running time of the algorithm in terms of seconds. To illustrate the quality of the results obtained by the AFSA and the compared algorithms, the mean relative error $(M R E)$ is also introduced. The relative error $(R E)$ is calculated as follows: $R E=(M S-L B) / L B \times 100 \%$, where $M S$ is the makespan obtained by the corresponding algorithm.

Table 4 Results of ten BRdata instances

\begin{tabular}{|c|c|c|c|c|c|c|c|c|c|c|c|c|c|c|c|c|c|c|}
\hline \multirow{2}{*}{ Instance } & \multirow{2}{*}{$n \times m$} & \multirow{2}{*}{$\mathrm{T}_{0}$} & \multirow{2}{*}{ Flex. } & \multirow{2}{*}{ (LB,UB) } & \multicolumn{3}{|c|}{$\mathrm{ABC}$} & \multicolumn{3}{|c|}{ HHS } & \multicolumn{4}{|c|}{ AFSA } & \multicolumn{4}{|c|}{ AFSA-ED } \\
\hline & & & & & $C_{m}$ & $A V$ & $S D$ & $C_{m}$ & $A V$ & $S D$ & $C_{m}$ & $A V$ & $S D$ & $T_{a}$ & $C_{m}$ & $A V$ & $S D$ & $T_{a}$ \\
\hline Mk01 & $10 \times 6$ & 55 & 2.09 & $(36,42)$ & 40 & 40.00 & 0.00 & 40 & 40.00 & 0.00 & 40 & 41.25 & 0.65 & 1.39 & 40 & 40.00 & 0.00 & 2.59 \\
\hline Mk02 & $10 \times 6$ & 58 & 4.01 & $(24,32)$ & 26 & 26.50 & 0.50 & 26 & 26.63 & 0.49 & 28 & 29.04 & 0.58 & 1.61 & 26 & 26.10 & 0.30 & 2.82 \\
\hline Mk03 & $15 \times 8$ & 150 & 3.01 & $(204,211)$ & 204 & 204.00 & 0.00 & 204 & 204.00 & 0.00 & 204 & 204.00 & 0.00 & 1.00 & 204 & 204.00 & 0.00 & 1.12 \\
\hline Mk04 & $15 \times 8$ & 90 & 1.91 & $(48,81)$ & 60 & 61.22 & 1.36 & 60 & 60.03 & 1.18 & 64 & 65.51 & 1.03 & 5.68 & 60 & 60.27 & 0.72 & 12.94 \\
\hline Mk05 & $15 \times 4$ & 106 & 1.71 & $(168,186)$ & 172 & 172.98 & 0.14 & 172 & 172.80 & 0.41 & 177 & 177.69 & 0.47 & 4.75 & 172 & 172.40 & 0.48 & 10.49 \\
\hline Mk06 & $10 \times 15$ & 150 & 3.27 & $(33,86)$ & 60 & 64.48 & 1.75 & 58 & 59.13 & 0.63 & 62 & 63.86 & 0.72 & 20.58 & 57 & 58.60 & 0.75 & 39.27 \\
\hline Mk07 & $20 \times 5$ & 100 & 2.83 & $(133,157)$ & 139 & 141.42 & 1.20 & 139 & 139.57 & 0.50 & 142 & 143.03 & 0.95 & 28.47 & 139 & 140.63 & 0.71 & 57.63 \\
\hline Mk08 & $20 \times 10$ & 225 & 1.43 & 523 & 523 & 523.00 & 0.00 & 523 & 523.00 & 0.00 & 523 & 523.00 & 0.00 & 1.04 & 523 & 523.00 & 0.00 & 2.14 \\
\hline Mk09 & $20 \times 10$ & 240 & 2.53 & $(299,369)$ & 307 & 308.76 & 1.63 & 307 & 307.00 & 0.00 & 310 & 310.75 & 0.54 & 4.06 & 307 & 307.13 & 0.43 & 9.40 \\
\hline Mk10 & $20 \times 15$ & 240 & 2.98 & $(165,296)$ & 208 & 212.84 & 2.43 & 205 & 211.13 & 2.37 & 213 & 214.91 & 1.37 & 50.44 & 201 & 201.93 & 1.06 & 104.10 \\
\hline
\end{tabular}

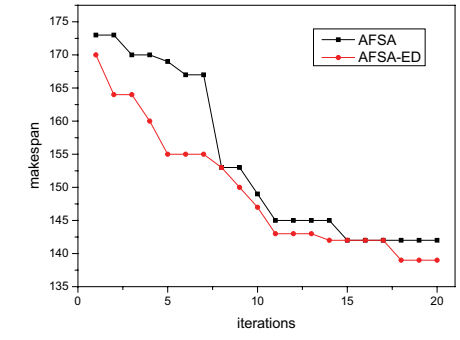

Fig.7 Convergence curves of MK07

\subsubsection{Influence of proposed EDA process}

To investigate the influence of proposed EDA process for preying behavior, the experiments on the BRdata are conducted by implementing the AFSA-ED and the AFSA without EDA, respectively. The parameters in the AFSA are taken as the same in AFSA-ED, and the two algorithms runs 30 independent times for each instance. The results are given in Table 4. Compared with AFSA, AFSA-ED outperforms AFSA in all 10 instances for the average makespan and the best makespan, and the $S D$ values obtained by AFSA-ED are relative smaller than AFSA for most of the instances. However, the overall computation time of the AFSAED is slightly longer than AFSA because of the EDA process. Fig. 7 and Fig. 8 show the convergence curves in solving MK7 and MK10 by AFSA-ED and AFSA respectively. The experiment results show the validity of the proposed EDA process for preying behavior.

\subsubsection{Results of BRdata problems}

The AFSA-ED is first tested on ten instances of BRdata. Meanwhile, we make a comparison with $\mathrm{ABC}$ algorithm by Wang et al. ${ }^{39}$ and HHS algorithm by Yuan et al. ${ }^{40}$. These results are also given in Table 4 . It can be seen

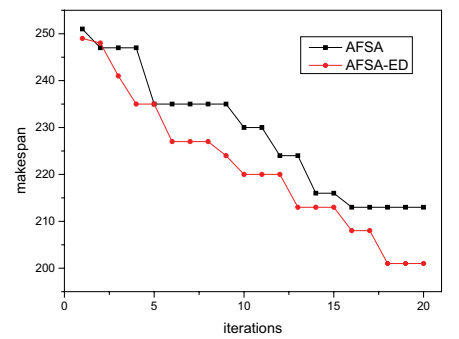

Fig. 8 Convergence curves of MK10

that AFSA-ED, ABC and HHS obtain the same best result for instances Mk01-Mk05, and Mk07-Mk09. For Mk06 and Mk10, AFSA-ED obtains the values of 57 and 201, respectively. On the other hand, the $\mathrm{ABC}$ obtains the best values of 60 and 208, respectively, and HHS obtains the best values of 58 and 205, respectively. Compared with $\mathrm{ABC}$, AFSA-ED outperforms $\mathrm{ABC}$ in all 10 instances for the average makespan, and the $S D$ values of AFSA-ED are relative smaller than ABC. In comparison with HHS, AFSA-ED outperforms HHS in 7 out 10 instances for the average makespan. It is worth noting that AFSA-ED can obtain average value of 201.93 and $S D$ value of 1.06 for instance $\mathrm{Mk} 10$, respectively. On the other hand, the HHS can obtain average value of 211.13 and $S D$ value of 2.37, respectively.

In addition, Table 5 gives a detailed comparison in terms of the MRE of the best value and the MRE of average value. We compare AFSA-ED with the PVNS of Yazdani et al. ${ }^{41}$, the CDDS of Ben Hmida et al. ${ }^{37}$, the BEDA of Wang et al. ${ }^{42}$, the ABC and the HHS. BKS represents the best known solution ever reported in the literature for each instance. It can be seen that AFSAED finds 9 best known solutions for 10 instances. The AFSA-ED obtains the MRE of the best value which is equal to $14.85 \%$, while PVNS, CDDS, ABC, BDEA, 
and HHS are $16.43 \%, 14.98 \%, 16.19 \%, 16.07 \%$, and $15.40 \%$, respectively. For the $M R E$ of average value obtained, AFSA-ED generates $15.64 \%$, faced to $16.48 \%$ for HHS, $17.01 \%$ for PVNS, $18.55 \%$ for $\mathrm{ABC}$, and $19.24 \%$ for BDEA. However, faced to $15.34 \%$ for the CDDS algorithm, the AFSA-ED Obtains a higher MRE. The Gantt chart of solution of MK06 obtained by AFSA-ED is showed in Fig.9.

To determine the statistical differences between the AFSA-ED and the compared algorithms, the Friedman test and Holm procedure are conducted. The results are presented in Table 6. It can be seen from the Friedman test results that the differences among the six algorithms are statistically relevant with $98 \%$ certainty. The AFSAED obtains the best overall rank. The holm procedure shows that the AFSA-ED obtains better results than the compared five algorithms, and the differences are statistically relevant with $97 \%, 66 \%, 81 \%, 86 \%$, and $67 \%$ certainty, respectively.

Table 5 Comparison between AFSA-ED and several existing algorithms on BRdata

\begin{tabular}{cccccccc}
\hline \multirow{2}{*}{ Instance } & \multirow{2}{*}{$\mathrm{BKS}$} & \multicolumn{2}{c}{ AFSA-ED } & \multicolumn{2}{c}{ PVNS } & \multicolumn{2}{c}{ CDDS } \\
\cline { 3 - 8 } & & $C_{m}(A V)$ & \multicolumn{1}{c}{$M R E$} & $C_{m}(A V)$ & $M R E$ & $C_{m}(A V)$ & $M R E$ \\
\hline Mk01 & 40 & $40(40)$ & $11.11(11.11)$ & $40(40)$ & $11.11(11.11)$ & $40(40)$ & $11.11(11.11)$ \\
Mk02 & 26 & $26(26.10)$ & $8.33(8.75)$ & $26(26.04)$ & $8.33(8.50)$ & $26(26)$ & $8.33(8.33)$ \\
Mk03 & 204 & $204(204)$ & $0.00(0.00)$ & $204(204)$ & $0.00(0.00)$ & $204(204)$ & $0.00(0.00)$ \\
Mk04 & 60 & $60(60.27)$ & $25.00(25.56)$ & $60(60.60)$ & $25.00(26.25)$ & $60(60)$ & $25.00(25.00)$ \\
Mk05 & 172 & $172(172.40)$ & $2.38(2.62)$ & $173(173)$ & $2.98(2.98)$ & $173(173.5)$ & $2.98(3.27)$ \\
Mk06 & 57 & $57(58.60)$ & $72.73(77.57)$ & $60(61)$ & $81.82(84.85)$ & $58(59)$ & $75.76(78.79)$ \\
Mk07 & 139 & $139(140.63)$ & $4.51(5.73)$ & $141(141.2)$ & $6.02(6.17)$ & $139(139)$ & $4.51(4.51)$ \\
Mk08 & 523 & $523(523)$ & $0.00(0.00)$ & $523(523)$ & $0.00(0.00)$ & $523(523)$ & $0.00(0.00)$ \\
Mk09 & 307 & $307(307.13)$ & $2.68(2.72)$ & $308(308.8)$ & $3.01(3.28)$ & $307(307)$ & $2.68(2.68)$ \\
Mk10 & 197 & $201(201.93)$ & $21.82(22.38)$ & $208(209.4)$ & $26.06(26.91)$ & $197(197.75)$ & $19.39(19.85)$ \\
MRE & & $14.85(15.64)$ & & $16.43(17.01)$ & & $14.98(15.34)$ & \\
\hline
\end{tabular}

Table 5 (Continued.) Comparison between AFSA-ED and several existing algorithms on BRdata

\begin{tabular}{cccccccc}
\hline \multirow{2}{*}{ Instance } & \multirow{2}{*}{$\mathrm{BKS}$} & \multicolumn{2}{c}{$\mathrm{ABC}$} & \multicolumn{2}{c}{ BEDA } & \multicolumn{2}{c}{ HHS } \\
\cline { 3 - 7 } & & $C_{m}(A V)$ & $M R E$ & $C_{m}(A V)$ & $M R E$ & $C_{m}(A V)$ & $M R E$ \\
\hline Mk01 & 40 & $40(40)$ & $11.11(11.11)$ & $40(41.02)$ & $11.11(13.94)$ & $40(40)$ & $11.11(11.11)$ \\
Mk02 & 26 & $26(26.50)$ & $8.33(10.24)$ & $26(27.25)$ & $8.33(13.54)$ & $26(26.63)$ & $8.33(10.96)$ \\
Mk03 & 204 & $204(204)$ & $0.00(0.00)$ & $204(204)$ & $0.00(0.00)$ & $204(204)$ & $0.00(0.00)$ \\
Mk04 & 60 & $60(61.22)$ & $25.00(27.54)$ & $60(63.69)$ & $25.00(32.69)$ & $60(60.03)$ & $25.00(25.06)$ \\
Mk05 & 172 & $172(172.98)$ & $2.38(2.96)$ & $173(173.38)$ & $2.98(3.20)$ & $172(172.80)$ & $2.38(2.86)$ \\
Mk06 & 57 & $60(64.48)$ & $81.82(95.39)$ & $60(62.83)$ & $81.82(90.39)$ & $58(59.13)$ & $75.76(79.18)$ \\
Mk07 & 139 & $139(141.42)$ & $4.51(6.33)$ & $139(141.55)$ & $4.51(6.43)$ & $139(139.57)$ & $4.51(4.94)$ \\
Mk08 & 523 & $523(523)$ & $0.00(0.00)$ & $523(523)$ & $0.00(0.00)$ & $523(523)$ & $0.00(0.00)$ \\
Mk09 & 307 & $307(308.76)$ & $2.68(2.93)$ & $307(310.35)$ & $2.68(3.80)$ & $307(307)$ & $2.68(2.68)$ \\
Mk10 & 197 & $208(212.84)$ & $26.06(28.99)$ & $206(211.92)$ & $24.85(28.44)$ & $205(211.13)$ & $24.24(27.96)$ \\
MRE & & $16.19(18.55)$ & & $16.07(19.24)$ & & $15.40(16.48)$ & \\
\hline
\end{tabular}

Table 6 Friedman test and Holm procedure of different algorithms

\begin{tabular}{|c|c|c|c|c|c|c|c|}
\hline \multicolumn{5}{|c|}{ Friedman test } & \multicolumn{3}{|c|}{ Holm procedure } \\
\hline Algorithm & Rank & $\chi^{2}$ & $1-p$ value & Diff.? & Algorithm & $Z$ & $1-p$ value \\
\hline AFSA-ED & 2.85 & \multirow{6}{*}{14.42} & \multirow{6}{*}{0.98} & \multirow{6}{*}{ Yes } & - & - & -2 \\
\hline PVNS & 4.50 & & & & AFSA-ED v.s. PVNS & 1.97 & 0.98 \\
\hline CDDS & 3.20 & & & & AFSA-ED v.s. CDDS & 0.41 & 0.66 \\
\hline $\mathrm{ABC}$ & 3.60 & & & & AFSA-ED v.s. ABC & 0.89 & 0.81 \\
\hline BEDA & 3.75 & & & & AFSA-ED v.s. BEDA & 1.07 & 0.86 \\
\hline HHS & 3.10 & & & & AFSA-ED v.s. HHS & 0.29 & 0.67 \\
\hline
\end{tabular}

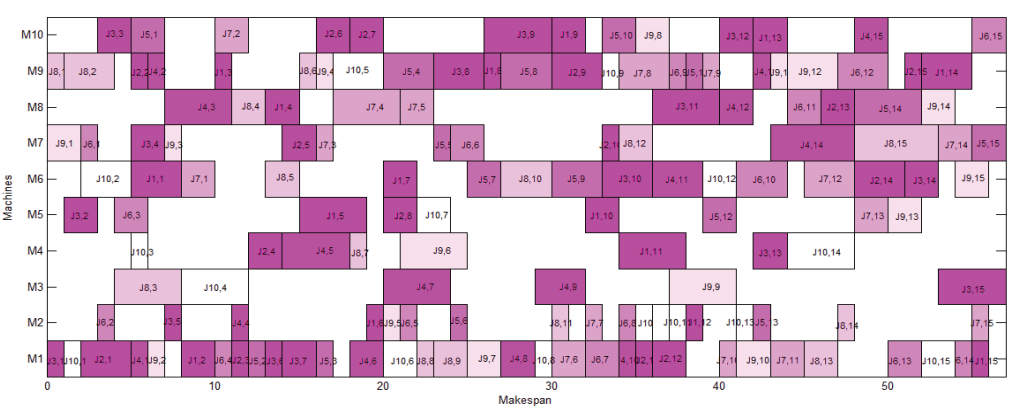

Fig.9 Gantt chart of solution of MK06 obtained by AFSA-ED (makespan=57) 


\subsubsection{Results of BCdata problems}

In this subsection, we carry out experiments on 21 instances of BCdata. The detail computational results are reported and compared with the CDDS and the HDE-N $\mathrm{N}_{1}$ of Yuan et al. ${ }^{43}$ in Table 7. As can be seen from Table 7, AFSA-ED outperforms CDDS and HDE-
$\mathrm{N}_{1}$ in 10 out of 21 instances. For the cases of seti5xxx and seti5xyz, CDDS obtains the best results of three algorithms, while AFSA-ED obtains better results than HDE-N ${ }_{1}$. The average values and $S D$ values of AFSAED are better than HDE-N $N_{1}$. AFSA-ED offers the comparable results with HDE- $\mathrm{N}_{2}$ while being faster than HDE-N ${ }_{2}$.

Table 7 Comparison of AFSA-ED with CDDS and HDE-N1 on BCdata

\begin{tabular}{|c|c|c|c|c|c|c|c|c|c|c|c|c|c|c|c|c|c|}
\hline \multirow{2}{*}{ Instance } & \multirow{2}{*}{$\begin{array}{l}\mathbf{n} \\
\times \mathbf{m} \\
\end{array}$} & \multirow{2}{*}{ Flex } & \multirow{2}{*}{ (LB,UB) } & \multicolumn{2}{|c|}{ CDDS } & \multicolumn{4}{|c|}{$\mathrm{HDE} \mathrm{N}_{1}$} & \multicolumn{4}{|c|}{ HDE-N ${ }_{2}$} & \multicolumn{4}{|c|}{ AFSA-ED } \\
\hline & & & & $C_{m}$ & $A V$ & $C_{m}$ & $A V$ & $S D$ & $T_{a}(s)$ & $C_{m}$ & $A V$ & $S D$ & $T_{a}(s)$ & $C_{m}$ & $A V$ & $S D$ & $T_{a}(s)$ \\
\hline $0 x$ & $10 \times 11$ & 1.10 & $(655$ & 918 & 918 & 918 & 922.86 & 6.11 & 21.43 & 918 & 918.58 & 2.20 & 179.22 & 918 & 18.00 & 0.00 & 23.47 \\
\hline $10 \mathrm{xx}$ & $10 \times 12$ & 1.20 & $(655,929)$ & 918 & 918 & 918 & 922.04 & 6.31 & 21.70 & 918 & 918.38 & 1.90 & 179.84 & 918 & 918.53 & 1.35 & 20.91 \\
\hline $\operatorname{mt10xxx}$ & $10 \times 13$ & 1.30 & $(655,936)$ & 918 & 918 & 918 & 919.94 & 3.96 & 23.05 & 918 & 918.00 & 0.00 & 179.39 & 918 & 18.17 & 0.37 & 25.38 \\
\hline $10 x y$ & $10 \times 12$ & 1.20 & $(655$ & 906 & 906 & 905 & 906.52 & 1.09 & 22.51 & 905 & 905.56 & 0.79 & 169.77 & 905 & 905.23 & 0.55 & 22.80 \\
\hline t10xyz & $10 \times 13$ & 1.30 & $(655$ & 849 & 850.5 & 847 & 856.80 & 3.99 & 21.79 & 847 & 851.14 & 4.65 & 160.24 & 847 & 851.07 & 2.93 & 24.63 \\
\hline $\mathrm{t} 10 \mathrm{c} 1$ & $10 \times 11$ & 1.10 & $(655,927)$ & 928 & 928.5 & 927 & 928.92 & 1.96 & 21.07 & 927 & 927.72 & 0.45 & 174.19 & 927 & 927.20 & 0.47 & 23.35 \\
\hline $\mathrm{mt} 10 \mathrm{cc}$ & $10 \times 12$ & 1.20 & $(655$, & 910 & 910.75 & 910 & 913.92 & 3.40 & 21.00 & 908 & 910.60 & 2.40 & 165.61 & 908 & 908.40 & 1.13 & 40.86 \\
\hline setb4x & $15 \times 11$ & 1.10 & $(846$ & 925 & 925 & 925 & 931.50 & 2.48 & 33.04 & 925 & 925.82 & 2.11 & 338.30 & 925 & 925.83 & 1.79 & 35.89 \\
\hline & $15 \times 12$ & 1.20 & $(84$ & 925 & 925 & 925 & 930.38 & 3.29 & 29.76 & 925 & 925.64 & 1.98 & & 925 & 925.56 & 1.72 & 38.64 \\
\hline & $15 \times 13$ & 1.3 & & 925 & 925 & 925 & 931.42 & 3.59 & & 925 & & 1.68 & & 925 & 925.60 & 1.45 & 36.42 \\
\hline $4 x y$ & $15 \times 12$ & 1.20 & & 916 & 916 & 910 & 921.38 & 4.44 & 31.13 & 910 & 914.00 & 3.50 & & 910 & 913.90 & 2.70 & 36.50 \\
\hline setb4xyz & $15 \times 13$ & 1.30 & $(83$ & 905 & 906.5 & 905 & 913.40 & 4.21 & 30.39 & 903 & 905.28 & 1.16 & & 903 & 904.06 & 0.85 & 76.28 \\
\hline setb $4 c 9$ & $15 \times 11$ & 1.10 & $(85$ & 919 & 919 & 914 & 919.32 & 2.87 & 32.19 & 914 & 917.12 & 2.52 & 313.02 & 914 & 916.26 & 2.17 & 64.27 \\
\hline Sctutec & $15 \times 12$ & 1.20 & & 909 & 910.5 & 909 & 58 & 3.81 & 32.00 & 907 & 58 & 1.89 & & 907 & 00 & 1.13 & 83.61 \\
\hline seti5x & $15 \times 16$ & 1.07 & $(95$ & 1201 & 1201.5 & 1204 & 5.48 & 5.36 & 73.20 & 1200 & 12 & 3.43 & 11 & 1198 & 1202.95 & 2.64 & 153.82 \\
\hline setisxx & $15 \times 17$ & 1.13 & 204) & 1199 & 1199 & 1202 & 1205.66 & 2.56 & 72.52 & 1197 & 120 & 2.02 & 10 & 1198 & 1201.23 & 1.99 & 93.12 \\
\hline seti5xxx & $15 \times 18$ & 1.20 & $(955,1213)$ & 1197 & 1197.5 & 1202 & 1206.10 & 3.18 & 72.07 & 1197 & 1202.26 & 2.37 & 1087.12 & 1197 & 1202.63 & 2.24 & 97.86 \\
\hline seti5xy & $15 \times 17$ & 1.13 & $(955,1148)$ & 1136 & 1138 & 1138 & 1146.86 & 5.04 & 78.98 & 1136 & 1137.98 & 2.82 & 1250.62 & 1136 & 1138.13 & 1.98 & 142.65 \\
\hline seti5xyz & $15 \times 18$ & 1.20 & $(955,1127)$ & 1125 & 1125.3 & 1130 & 1137.44 & 3.42 & 80.85 & 1125 & 1129.76 & 2.44 & 1244.22 & 1125 & 1129.53 & 1.76 & 405.74 \\
\hline $5 \mathrm{c} 12$ & $15 \times 16$ & 1.07 & $(1027,1185)$ & 1174 & 1174.5 & 1175 & 1182.54 & 7.62 & 69.06 & 1171 & 1175.42 & 1.63 & 1141.43 & 1174 & 1174.67 & 1.02 & 313.40 \\
\hline seti5cc & $15 \times 17$ & 1.13 & $(955,1136)$ & 1136 & 1137 & 1137 & 1145.62 & 5.58 & 78.83 & 1136 & 1137.76 & 2.48 & 1222.53 & 1136 & 1137.73 & 1.60 & 324.07 \\
\hline
\end{tabular}

Table 8 lists the best makespan and the $M R E$ of AFSA-ED, TSBM ${ }^{2} h$ by Bozejko et al. ${ }^{13}, \mathrm{CDDS}^{37}$, IFS by Oddi et al. ${ }^{44}$, HDE-N ${ }_{1}$ and HDE-N $\mathrm{N}_{2}$ by Yuan et al. ${ }^{43}$. For the IFS algorithm, its performance depends on the relaxing factor $\gamma$, the table lists the results obtained by running IFS with $\gamma$ ranging from 0.2 to 0.7 , respectively. The difference between $\mathrm{HDE}_{1} \mathrm{~N}_{1}$ and $\mathrm{HDE}-\mathrm{N}_{2}$ is neighborhood structure. Moreover, $\mathrm{HDE}-\mathrm{N}_{2}$ is more effective than HDE- $N_{1}$, but its computational time is much longer than HDE-N ${ }_{1}$. From Table 8 , as for the best makespan obtained, AFSA-ED obtains $85 \%$ of best known solutions, while $\mathrm{TSBM}^{2} \mathrm{~h}$ obtains $81 \%$, CDDS obtains $52 \%$, IFS obtains $47 \%$, HDE-N $\mathrm{N}_{1}$ obtains $52 \%$, and $\mathrm{HDE}-\mathrm{N}_{2}$ obtains $95 \%$. It can be seen that HDE-N outperforms AFSA-ED on two instances (seti5xxx, seti5c12), while AFSA-ED outperforms HDE-N $\mathrm{N}_{2}$ on one instance (seti5x). In particular, the $M R E$ of best makespan of AFSA is $22.39 \%$, faced to $22.45 \%$ for
$\mathrm{TSBM}^{2} \mathrm{~h}, 22.54 \%$ for CDDS, $22.55 \%$ for $\mathrm{HDE}-\mathrm{N}_{1}$, $23.09 \%$ for IFS $(\gamma=0.7)$, and $22.39 \%$ for HDE-N ${ }^{2}$. We note that AFSA-ED performs better than the $\mathrm{TSBM}^{2} \mathrm{~h}$, CDDS, IFS and HDE- $\mathrm{N}_{1}$, while the same with HDE- $\mathrm{N}_{2}$. However, the HDE- $\mathrm{N}_{2}$ tends to spend long time for finding best solutions. To determine the statistical differences among the AFSA-ED and the compared algorithms, the Friedman test and Holm procedure are also conducted. The results are presented in Table 9. It can be seen that the differences among the six algorithms are statistically relevant with $100 \%$ certainty. The AFSA-ED and the HDE-N $\mathrm{N}_{2}$ obtain the best overall rank. The Holm procedure shows that the AFSA-ED obtains the same results with the HDE- $\mathrm{N}_{2}$. The Holm procedure also shows that the AFSA-ED obtains better results than other four algorithms, and the differences are statistically relevant with $62 \%, 94 \%, 100 \%$, and $94 \%$, respectively. 
Table 8 Comparison of results using different algorithms (AFSA-ED, TSBM ${ }^{2} h$, CDDS, IFS, HDE)

\begin{tabular}{|c|c|c|c|c|c|c|c|c|c|c|c|c|}
\hline \multirow{2}{*}{ Instance } & \multirow{2}{*}{ BKS } & \multirow{2}{*}{ AFSA-ED } & \multirow{2}{*}{$\mathrm{TSBM}^{2} \mathrm{~h}$} & \multirow{2}{*}{ CDDS } & \multicolumn{6}{|l|}{ IFS } & \multirow{2}{*}{ HDE-N 1} & \multirow{2}{*}{$\mathrm{HDE}-\mathrm{N}_{2}$} \\
\hline & & & & & 0.2 & 0.3 & 0.4 & 0.5 & 0.6 & 0.7 & & \\
\hline $\operatorname{mt10x}$ & 918 & 918 & 922 & 918 & 980 & 936 & 936 & 934 & 918 & 918 & 918 & 918 \\
\hline $\operatorname{mt10xx}$ & 918 & 918 & 918 & 918 & 936 & 929 & 936 & 933 & 918 & 926 & 918 & 918 \\
\hline $\operatorname{mt10xxx}$ & 918 & 918 & 918 & 918 & 936 & 929 & 936 & 926 & 926 & 926 & 918 & 918 \\
\hline mt10xy & 905 & 905 & 905 & 906 & 922 & 923 & 923 & 915 & 905 & 909 & 905 & 905 \\
\hline mt10xyz & 847 & 847 & 849 & 849 & 878 & 858 & 851 & 862 & 847 & 851 & 847 & 847 \\
\hline mt10c1 & 927 & 927 & 927 & 928 & 943 & 937 & 986 & 934 & 934 & 927 & 927 & 927 \\
\hline $\mathrm{mt} 10 \mathrm{cc}$ & 908 & 908 & 908 & 910 & 926 & 923 & 919 & 919 & 910 & 911 & 910 & 908 \\
\hline setb $4 x$ & 925 & 925 & 925 & 925 & 967 & 945 & 930 & 925 & 937 & 937 & 925 & 925 \\
\hline setb4xx & 925 & 925 & 925 & 925 & 966 & 931 & 933 & 925 & 937 & 929 & 925 & 925 \\
\hline setb4xxx & 925 & 925 & 925 & 925 & 941 & 930 & 950 & 950 & 942 & 935 & 925 & 925 \\
\hline $4 x y$ & 910 & 910 & 910 & 916 & 910 & 941 & 936 & 936 & 916 & 914 & 910 & 910 \\
\hline setb4xyz & 903 & 903 & 903 & 905 & 928 & 909 & 905 & 905 & 905 & 905 & 905 & 903 \\
\hline setb4c9 & 914 & 914 & 914 & 919 & 926 & 937 & 926 & 926 & 920 & 920 & 914 & 914 \\
\hline setb4cc & 907 & 907 & 907 & 909 & 929 & 917 & 907 & 914 & 907 & 909 & 909 & 907 \\
\hline seti5x & 1198 & 1198 & 1198 & 1201 & 1210 & 1199 & 1199 & 1205 & 1207 & 1209 & 1204 & 1200 \\
\hline seti5xx & 1197 & 1198 & 1197 & 1199 & 1216 & 1199 & 1205 & 1211 & 1207 & 1206 & 1202 & 1197 \\
\hline seti5xxx & 1197 & 1197 & 1197 & 1197 & 1205 & 1206 & 1206 & 1199 & 1206 & 1206 & 1202 & 1197 \\
\hline seti5xy & 1136 & 1136 & 1136 & 1136 & 1175 & 1171 & 1175 & 1166 & 1156 & 1148 & 1138 & 1136 \\
\hline seti5xyz & 1125 & 1125 & 1128 & 1125 & 1165 & 1149 & 1130 & 1134 & 1144 & 1131 & 1130 & 1125 \\
\hline seti5c12 & 1171 & 1174 & 1174 & 1174 & 1196 & 1209 & 1200 & 1198 & 1198 & 1175 & 1175 & 1171 \\
\hline seti5cc & 1136 & 1136 & 1136 & 1136 & 1177 & 1155 & 1162 & 1166 & 1138 & 1150 & 1137 & 1136 \\
\hline$M R E$ & & 22.39 & 22.45 & 22.54 & 25.48 & 24.25 & 24.44 & 23.96 & 23.28 & 23.09 & 22.55 & 22.39 \\
\hline
\end{tabular}

Table 9 Friedman test and Holm procedure of different algorithms on BCdata

\begin{tabular}{|c|c|c|c|c|c|c|c|}
\hline \multicolumn{5}{|c|}{ Friedman test } & \multicolumn{3}{|l|}{ Holm procedure } \\
\hline Algorithm & Rank & $\chi^{2}$ & $1-p$ value & Diff.? & Algorithm & $z$ & $1-p$ value \\
\hline AFSA-ED & 2.76 & \multirow{11}{*}{143.05} & \multirow{11}{*}{1.00} & \multirow{11}{*}{ Yes } & - & - & - \\
\hline $\mathrm{TSBM}^{2} \mathrm{~h}$ & 3.10 & & & & AFSA-ED v.s. TSBM ${ }^{2} h$ & 0.33 & 0.62 \\
\hline CDDS & 4.36 & & & & AFSA-ED v.s. CDDS & 1.56 & 0.94 \\
\hline IFS 0.2 & 9.79 & & & & AFSA-ED v.s. IFS 0.2 & 6.86 & 1.00 \\
\hline IFS 0.3 & 8.88 & & & & AFSA-ED v.s. IFS 0.3 & 5.97 & 1.00 \\
\hline IFS 0.4 & 8.45 & & & & AFSA-ED v.s. IFS 0.4 & 5.55 & 1.00 \\
\hline IFS 0.5 & 7.95 & & & & AFSA-ED v.s. IFS 0.5 & 5.07 & 1.00 \\
\hline IFS 0.6 & 6.71 & & & & AFSA-ED v.s. IFS 0.6 & 3.85 & 1.00 \\
\hline IFS 0.7 & 6.83 & & & & AFSA-ED v.s. IFS 0.7 & 3.97 & 1.00 \\
\hline HDE-N & 4.40 & & & & AFSA-ED v.s. HDE- $\mathrm{N}_{1}$ & 1.60 & 0.94 \\
\hline $\mathrm{HDE}_{-N_{2}}$ & 2.76 & & & & AFSA-ED v.s. HDE- $\mathrm{N}_{2}$ & 0.00 & 0.50 \\
\hline
\end{tabular}

Table 10 Comparison of MRE obtained by using AFSA-ED, CDDS and HDE-N ${ }_{1}$ on HUdata

\begin{tabular}{|c|c|c|c|c|c|c|c|c|c|c|}
\hline \multirow{2}{*}{ Instance } & \multirow{2}{*}{$n \times m$} & \multicolumn{3}{|c|}{ Edata $(\boldsymbol{F l e x}=1.15)$} & \multicolumn{3}{|c|}{ Rdata $($ Fle $\boldsymbol{x}=2.0)$} & \multicolumn{3}{|c|}{ Vdata $($ Fle $\boldsymbol{x} \in[2.5,7.5])$} \\
\hline & & CDDS & HDE-N ${ }_{1}$ & AFSA-ED & CDDS & HDE-N 1 & AFSA-ED & CDDS & HDE-N ${ }_{1}$ & AFSA-ED \\
\hline $\mathrm{mt} 06 / 10 / 20$ & $\begin{array}{c}6 \times 6 \\
10 \times 10 \\
20 \times 5\end{array}$ & $\begin{array}{c}\mathbf{0 . 0 0} \\
(0.05)\end{array}$ & $\begin{array}{c}0.05 \\
(0.13)\end{array}$ & $\begin{array}{c}\mathbf{0 . 0 0} \\
(0.08)\end{array}$ & $\begin{array}{c}\mathbf{0 . 3 4} \\
(0.47)\end{array}$ & $\begin{array}{c}\mathbf{0 . 3 4} \\
(0.45)\end{array}$ & $\begin{array}{c}\mathbf{0 . 3 4} \\
(0.39)\end{array}$ & $\begin{array}{c}\mathbf{0 . 0 0} \\
(0.00)\end{array}$ & $\begin{array}{c}\mathbf{0 . 0 0} \\
(0.01)\end{array}$ & $\begin{array}{c}\mathbf{0 . 0 0} \\
(0.00)\end{array}$ \\
\hline la01-la05 & $10 \times 5$ & $\begin{array}{c}\mathbf{0 . 0 0} \\
(0.73)\end{array}$ & $\begin{array}{c}\mathbf{0 . 0 0} \\
(0.00)\end{array}$ & $\begin{array}{c}\mathbf{0 . 0 0} \\
(0.00)\end{array}$ & $\begin{array}{c}0.11 \\
(0.28)\end{array}$ & $\begin{array}{c}0.11 \\
(0.31)\end{array}$ & $\begin{array}{c}\mathbf{0 . 0 8} \\
(0.22)\end{array}$ & $\begin{array}{c}0.13 \\
(0.23)\end{array}$ & $\begin{array}{c}\mathbf{0 . 0 4} \\
(0.19)\end{array}$ & $\begin{array}{c}0.09 \\
(0.20)\end{array}$ \\
\hline la06-la10 & $15 \times 5$ & $\begin{array}{c}\mathbf{0 . 0 0} \\
(0.19)\end{array}$ & $\begin{array}{c}\mathbf{0 . 0 0} \\
(0.10)\end{array}$ & $\begin{array}{c}\mathbf{0 . 0 0} \\
(0.06)\end{array}$ & $\begin{array}{c}0.03 \\
(0.19)\end{array}$ & $\begin{array}{c}0.05 \\
(0.10)\end{array}$ & $\begin{array}{c}\mathbf{0 . 0 2} \\
(0.15)\end{array}$ & $\begin{array}{c}\mathbf{0 . 0 0} \\
(0.00)\end{array}$ & $\begin{array}{c}0.03 \\
(0.10)\end{array}$ & $\begin{array}{c}0.07 \\
(0.11)\end{array}$ \\
\hline la11-la15 & $20 \times 5$ & $\begin{array}{c}\mathbf{0 . 2 9} \\
(1.12)\end{array}$ & $\begin{array}{c}\mathbf{0 . 2 9} \\
(0.29)\end{array}$ & $\begin{array}{c}\mathbf{0 . 2 9} \\
(0.29)\end{array}$ & $\begin{array}{c}0.02 \\
(0.37)\end{array}$ & $\begin{array}{c}\mathbf{0 . 0 0} \\
(0.02)\end{array}$ & $\begin{array}{c}\mathbf{0 . 0 0} \\
(0.22)\end{array}$ & $\begin{array}{c}\mathbf{0 . 0 0} \\
(0.00)\end{array}$ & $\begin{array}{c}\mathbf{0 . 0 0} \\
(0.01)\end{array}$ & $\begin{array}{c}\mathbf{0 . 0 0} \\
(0.00)\end{array}$ \\
\hline la16-la20 & $10 \times 10$ & $\begin{array}{c}0.49 \\
(1.15)\end{array}$ & $\begin{array}{c}\mathbf{0 . 0 2} \\
(0.48)\end{array}$ & $\begin{array}{c}0.04 \\
(0.45)\end{array}$ & $\begin{array}{c}1.64 \\
(1.90)\end{array}$ & $\begin{array}{c}1.64 \\
(1.69)\end{array}$ & $\begin{array}{c}\mathbf{1 . 6 4} \\
(1.65)\end{array}$ & $\begin{array}{c}\mathbf{0 . 0 0} \\
(0.00)\end{array}$ & $\begin{array}{c}\mathbf{0 . 0 0} \\
(0.00)\end{array}$ & $\begin{array}{c}\mathbf{0 . 0 0} \\
(0.00)\end{array}$ \\
\hline la21-la25 & $15 \times 10$ & $\begin{array}{c}5.70 \\
(6.27)\end{array}$ & $\begin{array}{c}5.82 \\
(6.41)\end{array}$ & $\begin{array}{c}\mathbf{5 . 6 6} \\
(6.35)\end{array}$ & $\begin{array}{c}3.82 \\
(4.26)\end{array}$ & $\begin{array}{c}3.73 \\
(4.57)\end{array}$ & $\begin{array}{c}\mathbf{3 . 6 8} \\
(4.46)\end{array}$ & $\begin{array}{c}\mathbf{0 . 7 0} \\
(1.01)\end{array}$ & $\begin{array}{c}1.63 \\
(2.15)\end{array}$ & $\begin{array}{c}1.40 \\
(1.89)\end{array}$ \\
\hline la26-la30 & $20 \times 10$ & $\begin{array}{c}3.96 \\
(4.84)\end{array}$ & $\begin{array}{c}3.89 \\
(4.71)\end{array}$ & $\begin{array}{c}\mathbf{3 . 7 8} \\
(4.69)\end{array}$ & $\begin{array}{c}\mathbf{0 . 6 6} \\
(0.98)\end{array}$ & $\begin{array}{c}1.04 \\
(1.41)\end{array}$ & $\begin{array}{c}0.97 \\
(0.84)\end{array}$ & $\begin{array}{c}0.11 \\
(0.19)\end{array}$ & $\begin{array}{c}0.42 \\
(0.63)\end{array}$ & $\begin{array}{c}\mathbf{0 . 0 9} \\
(0.17)\end{array}$ \\
\hline la31-la35 & $30 \times 10$ & $\begin{array}{c}0.42 \\
(0.83)\end{array}$ & $\begin{array}{c}0.50 \\
(0.59)\end{array}$ & $\begin{array}{c}\mathbf{0 . 3 7} \\
(0.55)\end{array}$ & $\begin{array}{c}0.22 \\
(0.41)\end{array}$ & $\begin{array}{c}0.22 \\
(0.33)\end{array}$ & $\begin{array}{c}\mathbf{0 . 1 8} \\
(0.33)\end{array}$ & $\begin{array}{c}\mathbf{0 . 0 2} \\
(0.04)\end{array}$ & $\begin{array}{c}0.12 \\
(0.18)\end{array}$ & $\begin{array}{c}0.07 \\
(0.12)\end{array}$ \\
\hline la36-la40 & $15 \times 15$ & $\begin{array}{c}9.10 \\
(9.88)\end{array}$ & $\begin{array}{c}9.63 \\
(10.43)\end{array}$ & $\begin{array}{l}9.64 \\
(9.95)\end{array}$ & $\begin{array}{c}4.85 \\
(4.97)\end{array}$ & $\begin{array}{c}3.98 \\
(4.92)\end{array}$ & $\begin{array}{c}\mathbf{3 . 8 9} \\
(4.88)\end{array}$ & $\begin{array}{c}\mathbf{0 . 0 0} \\
(0.00)\end{array}$ & $\begin{array}{c}\mathbf{0 . 0 0} \\
(0.01)\end{array}$ & $\begin{array}{c}\mathbf{0 . 0 0} \\
(0.00)\end{array}$ \\
\hline$M R E(\%)$ & & $\begin{array}{c}2.32 \\
(2.91) \\
\end{array}$ & $\begin{array}{c}2.35 \\
(2.68) \\
\end{array}$ & $\begin{array}{c}\mathbf{2 . 3 0} \\
(2.60) \\
\end{array}$ & $\begin{array}{c}1.34 \\
(1.59) \\
\end{array}$ & $\begin{array}{c}1.28 \\
(1.59) \\
\end{array}$ & $\begin{array}{c}1.24 \\
(1.51)\end{array}$ & $\begin{array}{c}\mathbf{0 . 1 2} \\
(0.16) \\
\end{array}$ & $\begin{array}{c}0.26 \\
(0.38) \\
\end{array}$ & $\begin{array}{c}0.20 \\
(0.29) \\
\end{array}$ \\
\hline
\end{tabular}




\subsubsection{Results of HUdata problems}

This subsection shows the results of AFSA-ED for HUdata benchmark instances. Table 10 presents the $M R E$ of the best makespan and average makespan obtained by AFSA-ED, CDDS and HDE-N $\mathrm{N}_{1}$. It can be seen that AFSA can obtain the MRE of best value of $2.30 \%, 1.24 \%$ and $0.20 \%$ for Edata, Rdata and Vdata, respectively, while CDDS can obtain the MRE of best value of $2.32 \%, 1.34 \%$ and $0.12 \%$, respectively, HED$\mathrm{N}_{1}$ can obtain the MRE of best value of $2.35 \%, 1.28 \%$ and $0.26 \%$, respectively. Compared with CDDS, AFSAED could obtain better results than CDDS for some instances of Edata (la16-la35), some instances of Rdata (la01-la15, la31-la40), and some instances of Vdata (la01-la05, la26-la30). For the MRE of best and average makespan, AFSA-ED outperforms CDDS for Edata and Rdata, while CDDS obtains the best result of three algorithms for Vdata. In comparison with $\mathrm{HDE}_{-} \mathrm{N}_{1}$, AFSA-ED can obtain better results than HDE for some instances of Edata (mt06/10/20, la21-35), some instances of Rdata (la01-10,1a21-40) and some instances of Rdata (la06-10,la21-35). For the MRE of best and average makespan, AFSA-ED outperforms $\mathrm{HDE}-\mathrm{N}_{1}$ for three datasets.

Table 11 MRE of the best makespan obtained by AFSA-ED and other known algorithms

\begin{tabular}{ccccccccccc}
\hline Dada set & $\boldsymbol{P}_{\boldsymbol{N u m}}$ & AFSA-ED & GA_Chen & TS & GA_Pezzella & CDDS & PVNS $_{\text {HHS }}$ HDE-N $_{1}$ & HDE-N $_{2}$ \\
\hline BRdata & 10 & 14.85 & 19.55 & 19.55 & 17.53 & 14.98 & 16.39 & 15.40 & 15.58 & 14.67 \\
BCdata & 21 & 22.39 & 38.64 & 38.64 & 29.56 & 22.54 & 26.66 & 22.89 & 22.55 & 22.39 \\
Hurink Edata & 43 & 2.30 & 5.59 & 2.17 & 6.00 & 2.32 & 3.86 & 2.67 & 2.35 & 2.11 \\
Hurink Rdata & 43 & 1.24 & 4.41 & 1.24 & 4.42 & 1.34 & 1.88 & 1.88 & 1.28 & 1.05 \\
Hurink Vdata & 43 & 0.20 & 2.59 & 0.095 & 2.04 & 0.12 & 0.42 & 0.39 & 0.26 & 0.080 \\
\hline
\end{tabular}

\subsection{Discussions}

In Table 11, we summarize the $M R E$ of the best makespan obtained by our algorithm and other known algorithm for BRdata set, BCdata set and HUdata set. The first column shows the name of data set, the second column shows the number of problems for each set, the following nine columns show the MRE of AFSA-ED, GA of Chen ${ }^{15}$, TS of Mastrolilli ${ }^{12}$, GA of Pezzella ${ }^{17}$, CDDS, PVNS, HHS, HDE-N ${ }_{1}$ and HDE-N ${ }_{2}$. As can be seen in Table 11, HDE- $\mathrm{N}_{2}$ achieves the state-of-the-art performance on three data sets. Except for HDE- $\mathrm{N}_{2}$, AFSA-ED outperforms the other seven algorithms on BRdata and BCdata; it outperforms GA_Chen, GA_Pezzella, PVNS and HHS on three sub-data sets of HUdata, and CDDS on Edata and Rdata. TS works better than AFSA-ED on the HUdata, and CDDS works better than AFSA-ED on Vdata. In particular, HDE- $\mathrm{N}_{2}$ is a time consuming algorithm, in which the computational time is about 5 times longer than AFSAED as shown in Table 7. In conclusion, the results show that the AFSA-ED is an effective algorithm for FJSP.

\section{Conclusion}

In this paper, an efficient artificial fish swarm algorithm with estimation of distribution was proposed for solving the flexible job-shop scheduling problem with the criterion to minimize the makespan. Considering the interaction of two sub-problems, we propose the pre- principle and post-principle arranging mechanism to adjust machine assignment and operation sequence with different orders. For improving the global exploration of algorithm, we modify preying behavior with estimation of distribution and embed attracting behavior to the algorithm. To balance the exploration and exploitation, the critical path based local search was used. The proposed algorithm is tested on 160 well known benchmark instances and 121 best known solutions are found. The computational results and comparisons demonstrate that the proposed AFSA-ED outperforms several existing algorithms and is especially effective for the FJSP. In the future research, the AFSA could be used in multi-objective FJSP or many real world manufacturing systems.

\section{Acknowledgements}

The authors would like to thank the support of the National Natural Science Foundation of China (61572104, 61103146, 61402076, 61502072), Startup Fund for the Doctoral Program of Liaoning Province (20141023), the Fundamental Research Funds for the Central Universities (DUT15QY26, DUT15RC(3)088), and the project of the Key Laboratory of Symbolic Computation and Knowledge Engineering in Jilin University (93K172016K11). 


\section{References}

1. M.R. Garey, D.S. Johnson, and R. Sethi, The complexity of flow shop and job shop scheduling, Math. Oper. Res. 1(2) (1976) 117-129.

2. P. Bruker, and R. Schlie, Job-shop scheduling with multipurpose machines, Computing. 45(4) (1991) 369-375.

3. M. Singer and M. Pinedo, A computational study of branch and bound techniques for minimizing the total weighted tardiness in job shops, IIE trans. 30(2) (1998) 109-118.

4. N. Zribi, I. Kacem, A.E. Kamel, and P. Borne, Assignment and scheduling in flexible job-shops by hierarchical optimization, IEEE T. Syst. Man Cy. C. 37(4) (2007) 652-661.

5. J.B. Yang, GA-based discrete dynamic programming approach for scheduling in FMS environments, IEEE T. Syst. Man. Cy. B. 31(5) (2001) 824-835.

6. C.R. Scrich, V.A. Armentano, and M. Laguna, Tardiness minimization in a flexible job shop: a tabu search approach, J. Intell. Manuf. 15(1) (2004) 103-115.

7. B. Barzegar, H. Motameni, and H. Bozorgi, Solving flexible job-shop scheduling problem using gravitational search algorithm and colored Petri net, J. Appl. Math. 9 (2012) 701-708.

8. K. Kianfar, S.M.T. Fatemi Ghomi, and B. Karimi, New dispatching rules to minimize rejection and tardiness costs in a dynamic flexible flow shop, Int. J. Adv. Manuf. Technol. 45(7-8) (2009) 759-771.

9. M. Pinedo and M. Singer, A shifting bottleneck heuristic for minimizing the total weighted tardiness in a job shop, Nav. Res. Log, 46(1) (1999) 1-17.

10. C.A. Kaskavelis and M.C. Caramanis, Efficient Lagrangian relaxation algorithms for industry size jobshop scheduling problems, IIE transactions. 30(11) (1998) 1085-1097.

11. P. Brandimarte, Routing and scheduling in a flexible job shop by tabu search, Ann. Oper. Res, 41(3) (1993) 157183.

12. M. Mastrolilli and L. Gambardella, Effective neighbourhood functions for the flexible job shop problem. J. Scheduling. 3(1) (2000), 3-20.

13. W. Bozejko, M. Uchronski, and M. Wodecki, Parallel hybrid metaheuristics for the flexible job shop problem, Comput. Ind. Eng. 59(2) (2010) 323-333.

14. J. Li, Q. Pan, P. Suganthan, and T. Chua, A hybrid tabu search algorithm with an efficient neighborhood structure for the flexiblejob shop scheduling problem, Int. J. Adv. Manuf. Technol. 52(5-8) (2011) 683-697.

15. H. Chen, J. Ihlow, and C. Lehmann, A genetic algorithm for flexible job-shop scheduling, Proc. of the International Conference on Robotics and Automation, (Detroit, Michigan, 1999), pp.1120-1125.

16. I. Kacem, S. Hammadi, and P. Borne, Approach by localization and multiobjective evolutionary optimization for flexible job-shop scheduling problems, IEEE T. Syst. Man. Cy. C. 32(1) (2002) 1-13.
17. F. Pezzella, G. Morganti, and G. Ciaschetti, A genetic algorithm for the flexible job-shop scheduling problem, Comput. Oper. Res. 35(10) (2008) 3202-3212.

18. J. Gao, L. Sun, and M. Gen, A hybrid genetic and variable neighborhood descent algorithm for flexiblejob shop scheduling problems, Comput. Oper. Res. 35(9) (2008) 2892-2907.

19. N.M. Najid, S. Dauzere-Peres, and A. Zaidat, A modified simulated annealing method for Flexible Job Shop Scheduling Problem, IEEE Sys. Man. Cy. A. 5 (2002) 117-123.

20. P. Fattahi, A hybrid multi objective algorithm for flexible job shop scheduling, Int. J. Comput. Math. Sci. 3 (2009) 215-220.

21. N. Shivasankaran, P. Senthilkumar, and K. Venkatesh Raja K, Hybrid non-dominated sorting simulated annealing algorithm for flexible job shop scheduling problems, Proc. of the 48th Annual Convention of Computer Society (Visakhapatnam, India, 2014), vol.248, pp.101-107.

22. B.S. Girish and N. Jawahar, A particle swarm optimization algorithm for flexible job shop scheduling problem, Proc. of the IEEE International Conference on Automation Science and Engineering (Bangalore, India, 2009), pp.298-303.

23. G. Zhang, X. Shao, P. Li, and L. Gao, An effective hybrid particle swarm optimization algorithm for multiobjective flexible job-shop scheduling problem, Comput. Ind. Eng. 56(4) (2009) 1309- 1318.

24. X. Shao, W. Liu, Q. Liu, and C. Zhang, Hybrid discrete particle swarm optimization for multi-objective flexible job-shop scheduling problem, Int. J. Adv. Manuf. Technol. 67(9-12) (2013) 2885-2901.

25. B.Z. Yao, C.Y. Yang, J.J. Hu, J.B. Yao, and J. Sun, An improved ant colony optimization for flexible job shop scheduling problems, Adv. Sci. Lett. 4(6-7) (2011) 2127 2131.

26. S.K. Sim, K.T. Yeo, and W.H. Lee, An expert neural network system for dynamic job-shop scheduling, Int. J. Prod. Res. 32(8) (1994) 1759-1773.

27. A. Bagheri, M. Zandieh, I. Mahdavi, and M. Yazdani, An artificial immune algorithm for the flexible job-shop scheduling problem, Future Gener. Comp. Sy. 26(4) (2010) 533-541.

28. X.S. Han, Y.C. Liang, and Z.G. Li, An efficient genetic algorithm for optimization problems with timeconsuming fitness evaluation, Int. J. Comp. Meth.-Sing, 12(1) (2015) 1350106 1-24.

29. X. Li, Z. Shao, and J. Qian, An optimizing method based on autonomous animates: fish-swarm algorithm, Syst. Eng. Theory. Prac. 22(11) (2002): 32-38.

30. M. Neshat, G. Sepidnam, M. Sargolzaei, and A.N. Toosi, Artificial fish swarm algorithm: a survey of the state-ofthe-art, hybridization, combinatorial and indicative applications, Artif. Intell. Rev. 42(4) (2002) 965-997.

31. M. Jiang and K. Zhu, Multiobjective optimization by artificial fish swarm algorithm. Proc. of the IEEE 
International Conference on Automation Science and Engineering, (Trieste, Italy, 2011), pp.506-511.

32. K. Zhu and M. Jiang, The optimization of job shop scheduling problem based on artificial fish swarm algorithm with tabu search strategy, Proc. of International Workshop on Advanced Computational Intelligent (Hangzhou, China, 2013), pp.323-327.

33. S. He, N. Belacel, H. Hamam, and Y. Bouslimani, Fuzzy clustering with improved artificial fish swarm algorithm, Proc. of the Int. Joint Conf. on Computational Sciences and Optimization (Hainan, Sanya, China, 2009), vol.2, pp.317-321.

34. H.W. Ge, L. Sun, Y.C. Liang, and F. Qian, An effective PSO-and-AIS-based hybrid intelligent algorithm for jobshop scheduling, IEEE T. Syst. Man. Cy. A., 38(2) (2008) 358-368.

35. J.W. Barnes and J.B. Chambers, Flexible job shop scheduling by tabu search, Graduate program in operations research and industrial engineering, the University of Texas at Austin, Technical Report Series (1996), ORP96-09.

36. J. Hurink, B. Jurisch, and M. Thole, Tabu search for the job-shop scheduling problem with multi-purpose machines, OR. Spectrum. 15(4) (1994) 205-215.

37. A. Ben Hmida, M. Haouari, M.J. Huguet, and P. Lopez, Discrepancy search for the flexible job shop scheduling problem, Comput. Oper. Res. 37(12) (2010) 2192- 2201.
38. B. Jurisch, Scheduling jobs in shops with multi-purpose machines. Thesis PhD. Fachbereich Mathematik Informatik (Universitat Osnabruck, 1992).

39. L. Wang, G. Zhou, Y. Xu, S. Wang, and M. Liu, An effective artificial bee colony algorithm for the flexible job-shop scheduling problem, Int. J. Adv. Manuf. Technol. 60(1-4) (2012) 303-315.

40. Y. Yuan, H. Xu, and J. Yang, A hybrid harmony search algorithm for the flexible job shop scheduling problem, Appl. Soft. Comput. 13(7) (2013) 3259-3272.

41. M. Yazdani, M. Amiri, and M. Zandieh, Flexible jobshop scheduling with parallel variable neighborhood search algorithm, Expert Syst. Appl. 37(1) (2010) 678687.

42. L. Wang, S. Wang, Y. Xu, G. Zhou, and M. Liu, A bipopulation based estimation of distribution algorithm for the flexible job-shop scheduling problem, Comput. Ind. Eng. 62(4) (2012) 917-926.

43. Y. Yuan and H. Xu, Flexible job shop scheduling using hybrid differential evolution algorithms, Comput. Ind. Eng. 65(2) (2013) 246-260.

44. A. Oddi, R. Rasconi, A. Cesta, and S. Smith, Iterative flattening search for the flexible job shop scheduling problem, Proc. of the International Joint Conferences on Artificial Intelligence (Barcelona, Catalonia, Span, 2011), 22(3): 1991-1996. 